\title{
Gerst, Dominik, Maria Klessmann, und Hannes Krämer (Hrsg.) (2021): Grenzforschung: Handbuch für Wissenschaft und Studium
}

\author{
Baden-Baden: Nomos. 559 Seiten. 98,00€
}

\author{
Alexandra Berger $(\mathbb{D}$
}

Angenommen: 20. Dezember 2021 / Online publiziert: 11. Januar 2022

(C) Deutsche Vereinigung für Politikwissenschaft 2022

Die Grenzforschung in ihrer heutigen Form ist ein noch recht junger Forschungszweig, der in den letzten Dekaden insbesondere auch im deutschsprachigen Wissenschaftsraum einen deutlichen Aufschwung und eine Diversifizierung erlebt hat. So entstand das Desiderat eines deutschsprachigen Übersichtswerks über die Forschung zu Grenzen, welches nun durch das vorliegende Handbuch angegangen wird. Dabei ist die Politikwissenschaft eines der Fächer, die sich naturgemäß für das Studium von Grenze(n) und Grenzziehungsprozessen interessieren, wenn auch die dezidiert politikwissenschaftliche Grenzforschung erst einige Jahrzehnte alt ist (S. 13). Aus politologischer Sicht interessieren dabei insbesondere Strukturierungen politischer Ordnungen sowie Identitäten und damit gleichsam Inklusions- und Exklusionsfunktionen von Grenze(n).

Der Sammelband dient hier sowohl als Einführung als auch als Übersichtswerk und setzt sich das ambitionierte Ziel, die Erforschung von Grenze(n) in ihrer Breite und Tiefe abzubilden. Das Buch richtet sich dabei an fortgeschrittene Studierende und WissenschaftlerInnen unterschiedlichster disziplinärer Herkunft. Aus formaler Perspektive erwähnenswert ist das enthaltene Interview mit Chiara Brambilla, Didier Fassin und Sarah Green, welches die Grenzforschung fassbarer macht, aber gleichzeitig auch deren Multiperspektivität unterstreicht.

Für die Politikwissenschaft als eine der Mutterdisziplinen der Grenzforschung ist es insbesondere bedeutsam, die Erweiterung und Auffächerung der disziplinären Zugänge und damit auch der Konzeptionen von Grenze(n) zu verfolgen. „Bis in die 1990er-Jahre hatte sich die Grenzforschung überwiegend auf zwischenstaatliche,

\footnotetext{
Alexandra Berger $(\varangle)$

Seminar für Politikwissenschaft und Politikdidaktik, Europa-Universität Flensburg, Flensburg,

Deutschland

E-Mail: Alexandra.Berger@uni-flensburg.de

Ghent Institute for International and European Studies, Universität Gent, Gent, Belgien
} 
militärische, koloniale und politisch-institutionelle Grenzen konzentriert“ (S. 363). Indem der (National-)Staat somit als Bezugspunkt diente, folgte die Grenzforschung, zumindest implizit, einem methodologischen Nationalismus. Seitdem wurde dieser Forschungszweig jedoch thematisch sowie disziplinär erweitert und zeichnet sich nun durch ein breites, inklusives Grenzverständnis aus. So ist es den HerausgeberInnen des Sammelbandes ein Anliegen, den methodologischen Nationalismus in der Betrachtung von Grenzen zu überwinden und folglich Grenzen auch jenseits des (National-)Staats zu denken. Dabei sollen insbesondere nichtstaatliche Akteure und nichtstaatliche Grenzprozesse in den Blick genommen werden. „Aber auch heute greifen staatliche Grenzen auf entscheidende Weise in die Lebenswelten und Entfaltungsmöglichkeiten vieler Menschen ein“ (S. 285). Obwohl es ein wichtiger Beitrag der interdisziplinären Grenzwissenschaften ist, den Fokus auf politisch-räumliche Grenzen um andere Grenzprozesse sowie um den Aufbau und Abbau von Grenze(n) zu erweitern, kann der (National-)Staat in vielen Arbeiten als wichtiger Akteur oder zumindest als intervenierende Variable nicht außer Acht gelassen werden. Der Politikwissenschaft fiel und fällt hierbei insofern eine Schlüsselrolle zu, als es Concha Maria Höfler und Maria Klessmann folgend zwar äußerst relevant ist, „,dass sich (national-)staatliche Grenzen nicht losgelöst von soziosymbolischen Grenzen verstehen lassen“ (S. 346), jedoch gleichwohl der Blick auf „die Nation“ für das Verständnis von Grenzen oftmals wesentlich ist. Während das Handbuch also den methodologischen Nationalismus überwinden will, kommen die Grenzforschung und folglich auch das Buch nicht ohne die Betrachtung des Staates als zentralen Akteur aus. Insofern ist das Werk ein Ausdruck des ambivalenten Verhältnisses der Grenzforschung zum methodologischen Nationalismus und zur Perspektive auf politisch-territoriale Grenzen („borders“). Die Ambivalenz macht sich darin bemerkbar, dass einige Beiträge soziale Grenzziehungen (,boundaries“), also soziosymbolische, kulturelle, ästhetische, ethnisierte Grenzprozesse betrachten, während andere dann doch recht häufig „borders“ in den Blick nehmen (S. 352).

So lassen sich denn auch viele Beiträge disziplinär in einer weit verstandenen Politikwissenschaft verorten. Gleichwohl lädt die Multiplizität des Bandes dazu ein, aus dem Blickwinkel verschiedener Fächer kritisch über das Verhältnis von „border" und „boundary“ nachzudenken. Ohne in etatistische Fallen zu tappen, sollen staatliche Akteure und politisch-territoriale Grenzen dabei nicht außer Acht gelassen werden. Die Zusammenschau der einzelnen Kapitel des Sammelbandes trägt erheblich zu diesem Unterfangen bei. Im Zuge dessen leistet dieses erste deutschsprachige Übersichtswerk einen wichtigen Beitrag zur Genese der Grenzwissenschaften im deutschsprachigen Raum. Für die Politikwissenschaft bedeutet dies eine Ausweitung der in den Blick genommenen Grenzen, Grenzprozesse und Akteure. Als konkretes Beispiel kann hier Béatrice von Hirschhausens Konzept der „Phantomgrenzen“ (S. 175-189) dienen, welches den Blick auf gegenwärtige Diskontinuitäten lenkt, die auf historische Territorialgrenzen zurückzuführen sind. Beispielsweise zeugt das Wahlverhalten bei den Präsidentschaftswahlen in Polen noch stets von früheren polnischen Staatsgrenzen (S. 178).

Etwas schade ist, dass das Handbuch sich hauptsächlich auf die Bundesrepublik bzw. auf Europa konzentriert, obwohl doch Grenzziehungsprozesse außerhalb Europas viel empirisches Material für die Grenzforschung liefern. Im Zuge ihrer 
Betrachtung des Frontier-Begriffs lenken Conrad Schetter und Marie Müller-Koné den Blick dann auch auf Asien, Afrika und Nordamerika und betonen die kolonialen Ursprünge vieler Frontier-Konzeptionen, die oftmals auf die wirtschaftliche Aneignung der ,Wildnis“ zurückgehen (S. 240-253). Weitere Beiträge, die sich mit Erdteilen außerhalb Europas beschäftigen, fehlen jedoch.

Weiterhin fällt ins Auge, dass der Sammelband eine inhaltliche Leerstelle in Form der Coronapandemie aufweist. Im Januar 2021 erschienen, war es dem Band verständlicher- und redlicherweise nicht möglich, die Covid-19-Pandemie in der Grenzforschung zu rezipieren sowie deren Auswirkungen auf Grenze(n) detailliert zu reflektieren. Zwei Beiträge beschäftigen sich jedoch zumindest tentativ mit der Pandemie. Insofern hätte die Einleitung des Werks zum Beispiel durch die Pandemie entstehende Fragen für die Grenzforschung formulieren können. Andererseits wäre es möglich gewesen, die Covid-19-Pandemie explizit aus der Betrachtung auszuschließen. Zwischen diesen beiden Optionen wurde ein Mittelweg gewählt, der etwas unbedacht erscheint. Diese Vorgehensweise kann jedoch mit der zeitlichen Koinzidenz plausibel erklärt werden. Insofern ist der Sammelband inmitten einer für die Grenzforschung bedeutenden Zeit erschienen, in der sich Ereignisse überschlugen, die die kontinuierliche Relevanz dieses Forschungszweigs unterstreichen. 